\title{
Towards design optimization of high-pressure gasoline injectors using Genetic Algorithm coupled with Computational Fluid Dynamics (CFD)
}

\author{
Robin Hellmann*, Paul Jochmann ${ }^{1}$, Karl Georg Stapf ${ }^{1}$, Erik Schuenemann ${ }^{1}$, László Daróczy² \\ Dominique Thévenin ${ }^{2}$ \\ ${ }^{1}$ Robert Bosch GmbH, Schwieberdingen, Germany \\ ${ }^{2}$ The Laboratory of Fluid Dynamics and Technical Flows, Otto-von-Guericke-University, \\ Magdeburg, Germany \\ *Corresponding author: robin.hellmann@de.bosch.com
}

\begin{abstract}
The spray pattern of high-pressure multi-hole injectors as well as the atomization process are of uttermost importance regarding efficiency and emissions in gasoline combustion engines. Ensuring optimal homogenization while meeting the engine individual specifications regarding spray targeting and massflow is a crucial development goal. High effort is put on the layout of the nozzle seat to meet the engine requirements. Success is only possible with a deep knowledge of the influencing quantities, considering that many design parameters affect the inner nozzle flow. Based on this knowledge improvement in spray penetration length and atomization can be achieved.

In the current investigation a segment model of the injector is considered. A fully automated, highly parallelized workflow enables a systematic examination of the constrained design space with acceptable computational time. The CFD workflow is implemented in the OPtimization Algorithm Library++ (OPAL++) developed at the "Otto von Guericke" University of Magdeburg.

First, inner nozzle flow 3D-CFD calculations of two selected nozzle geometries are validated by comparison with shadowgraphy and Long-Distance-Microscope (LDM) measurements. Using these simulations, correlations between nozzle flow parameters and the key spray characteristics, serving as optimization objectives, are analyzed. Second, a Design-of-Experiment (DoE) is created to understand the interdependency between design variables and objectives. Based on the DoE, metamodels are constructed, validated, compared with each other and used for optimization. Afterwards, a direct 3D CFD-optimization is carried out for the nozzle geometry. It relies on a Genetic Algorithm in OPAL++ to identify the Pareto front of the multi-objective problem. Finally, the Pareto front is analyzed and conclusions are drawn for future research.
\end{abstract}

\section{Keywords}

Nozzle flow, High Pressure Injector, CFD, Metamodeling, Genetic Algorithm, Optimization

\section{Introduction}

In the recent years the demand for high power output and high efficiency combined with stricter emission regulations has led to a broad use of Gasoline Direct Injection (GDI) engines. To fulfill the engine individual requirements as well as the legislation emission regulations high development effort was put towards the injection system. Different fields of investigations cover system pressure, type and position of injector, injection timing as well as geometric studies of the key valve seat parameter, e.g. [17,22]. The latter are principally responsible for the primary spray characteristics like spray plume trajectory, spray plume cone angle, atomization and penetration. Further engine-specific demands, e.g., cylinder geometry, charge motion, mass flow rate, etc., require a flexible design. The designs of nozzle seat and spray hole are crucial for the development of current combustion systems [11,21].

Most published studies consider only a few designs obtained by changing a single parameter $[14,15,20]$. For multihole high pressure gasoline injectors the manufacturing process has reached a high degree of design flexibility, e.g., due to laser-drilled spray holes. A systematic exploration of this design space with manufactured injectors would be very expensive and time consuming. However, rising computational power together with more efficient simulation models opens the field for extensive design parameter studies; it is then possible to use genetic algorithms (GA) for design optimization. Optimization based on Computational Fluid Dynamics (abbreviated CFD-O) with the help of GA has been already successfully applied to various engineering problems, e.g., [7,8]. In the present paper the goal of the optimization is to achieve the best possible atomization and to lower the penetration length of a single plume by maximizing the Turbulent Kinetic Energy (TKE) and the spray opening angle $\beta$ at the spray hole outlet plane. For this purpose, four design variables are selected: spray hole length $s h_{l}$, spray hole conicity $s h_{c o n}$, counterbore diameter $c b_{d}$ and the wall thickness $w t$ of the valve seat.

Current state-of-the-art computational fluid dynamic (CFD) methods are capable to predict the massflow and spray characteristics with sufficient accuracy. In the present study idealized conditions (1 bar cylinder pressure with an incompressible and isothermal flow at $20^{\circ} \mathrm{C}$ ) are applied to the Reynolds-averaged Navier-Stokes (RANS) simulation method using an Eulerian multiphase treatment. The setup is first validated with Long Distance Microscope (LDM) and shadowgraphy measurements of a generic two-hole injector. Correlations between the two chosen objectives and the nozzle flow data from the simulations are identified. 
As 3D-CFD simulations of the inner nozzle flow are still computationally time-consuming, only a segment of the injector with a smaller computational domain is considered. A fully automatic workflow is set up consisting of geometry creation, meshing, definition and solving of the problem, postprocessing as well as coupling with the GA. Using OPAL++, approximation models can be considered, so that response surface models (RSM) are built on a design of experiments (DoE), like suggested in $[16,18,19]$. For each objective a RSM is built by a regression analysis and used to identify interrelationships between design variables and objectives. Furthermore, the RSM models are deployed to a multi-objective optimization process, which later is compared with the results of a pure CFD-based optimization.

\section{Geometry and Numerical Method}

Figure 1 shows the high pressure gasoline injector (left), the valve seat including the pintle (middle) and the simulation domain of a segment model (right). To better understand the interdependency between inner nozzle flow simulations and spray characteristics, measurements were performed with a purpose-built two-hole injector, each hole arranged at the same radius from the injector axis at $180^{\circ}$ circumferential spacing. The nozzle inclination angles $\alpha$, skew angle between vertical injector axis and spray hole axis, are $10^{\circ}$ - hereinafter referred to as "SH1" - and $50^{\circ}$ - hereinafter referred to as "SH2" - leading to two different sharp bends at the transition from the nozzle seat to the spray hole inlet. This region of transition influences the highly transient, non-homogeneous and turbulent inner nozzle flow. Therefore it is indispensable that the numerical model should reflect the flow characteristics of both holes. The remaining geometric parameter of the two holes are identical. The length-to-diameter ratio $s h_{l} / s h_{d}$ of the slightly diverging hole with conicity angle $s h_{c o n}$ is approximately 1 with a nozzle length $s h_{l}$ of order $200 \mu \mathrm{m}$. A cylindrical "counterbore" with length-to-diameter ratio $c b_{l} / c b_{d}$ of approximately 0.75 is attached to the nozzle. The wall thickness $w t$ of the valve seat is hence the sum of spray hole and counterbore length. Figure 2 depicts the parameter being investigated in the present study. Compared to production type multi-hole injectors the nozzle flow and spray plume interaction for the two-hole injector is, due to the distance, negligible [10]. The assumption of spray hole independency permits the analysis of a segment model representing one single hole from a five-hole injector. The simulation domain covers the smallest gap between pintle and valve seat, the spray hole with downstream counterbore and the cylinder volume, partly represented by an ambient domain ending after an axial expansion of $0.5 \mathrm{~mm}$, like depicted in Figure 1. All subsequent investigations do not include pintle motion as this phase is only about ten percent of the whole injection duration. Thus this impact on the penetration length is negligible for a typical homogeneous gasoline combustion process. Additionally limited computational effort required for CFD-O can be ensured. Hence, the transient simulations are performed for a static geometry with a pintle displacement at full lift.
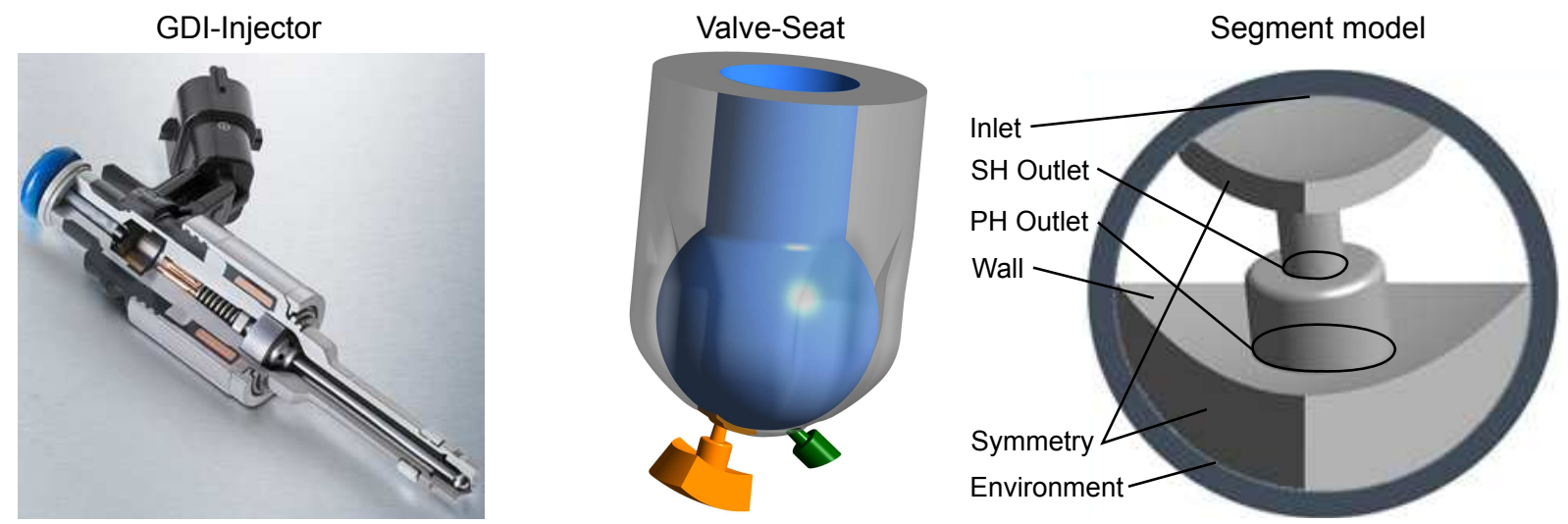

Figure 1. Simulation domain of segment model [23]

ANSYS CFX, a commercial CFD tool, is employed to simulate the two-component and two-phase flow (n-heptane, $\mathrm{n}$-heptane vapor, air) of the high-pressure injector. The three-fluid system is represented with a homogeneous model, an Eulerian-Eulerian method where all phases share a single velocity field [24]. Mass transfer between fuel and fuel vapor is considered with the use of the Rayleigh-Plesset cavitation model. Turbulence is treated with the RANS approach where flow variables are decomposed into mean and fluctuation terms. Stochastic effects of turbulence is represented through the two additional transport equations of the $k$ - $\omega$-SST turbulence model, used to close the system of partial differential RANS equations for mass and momentum conservation. Furthermore, the flow is considered as incompressible and isothermal at $293.15 \mathrm{~K}$. A total pressure of 200 bar serves as inlet boundary condition and an opening with a reference pressure of 1 bar is applied to the boundaries of the ambient domain. The sidewalls are treated as symmetry planes.

The simulation setup for validation covers a physical time of $0.125 \mathrm{~ms}$ with a timestep of $10^{-7}$ seconds. As the flow field has to develop from rest the analysis starts after 0.025 ms. ANSYS CFX uses the Finite Volume Method for spatial discretization so that a hexahedral mesh is generated with ANSYS Meshing. Mesh refinements in the regions of high pressure and velocity gradients, i.e., close to the walls, in the spray hole and counterbore, ensure an adequate solution of the complex 3D-flow. Results from a preliminary mesh study lead to the mesh consisting of roughly 3 million nodes with average axial cell size in the spray hole of $3.5 \mu \mathrm{m}$. 


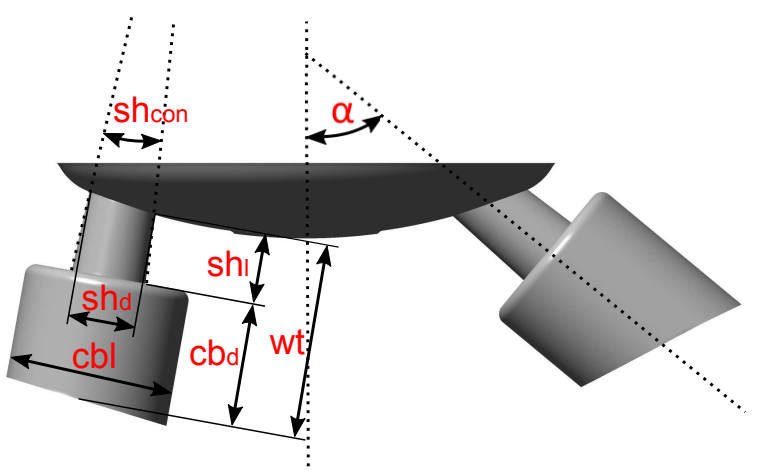

Figure 2. Geometry parameter

\section{Validation and Correlations}

To validate the simulation setup, the outflow and the spray in the nearfield of the injector are recorded with a LongDistance-Microscope (LDM) at $100 \mathrm{kHz}$ in a spray chamber. Additionally, shadowgraphy measurements at $25 \mathrm{kHz}$ visualize the penetration for a large image section of $124 \mathrm{~mm} \times 100 \mathrm{~mm}$.

Table 1. Simulation setup and measurement conditions

\begin{tabular}{r|ccccc}
\hline & $\begin{array}{c}\text { Pressure } \\
\text { difference }\end{array}$ & Fuel & Temperature & $\begin{array}{c}\text { Chamber } \\
\text { fluid }\end{array}$ & Duration \\
\hline Measurement & $200 \mathrm{bar}$ & n-heptane & $293.15 \mathrm{~K}$ & $\mathrm{~N}_{2}$ & $1.5 \mathrm{~ms}$ \\
Simulation & $200 \mathrm{bar}$ & n-heptane & $293.15 \mathrm{~K}$ & Air & $0.125 \mathrm{~ms}$
\end{tabular}

The conditions of the spray chamber compared to the simulation setup are given in Table 1. For safety reason the spray chamber is purged with $\mathrm{N}_{2}$ instead of air, as used in the simulation setup. For the spray chamber tests a realistic injection duration of $1.5 \mathrm{~ms}$ is chosen to ensure a static massflow in the middle of the injection sequence. First, shadowgraphy measurements (Figure 3) visualize the spray plume penetration with a frequency of $200 \mu \mathrm{s}$. The pintle motion at the beginning of injection results in a lower penetration for $\mathrm{SH} 2$, however, this effect levels out so that after $1000 \mu$ s the penetration length of both holes is approximately equal.

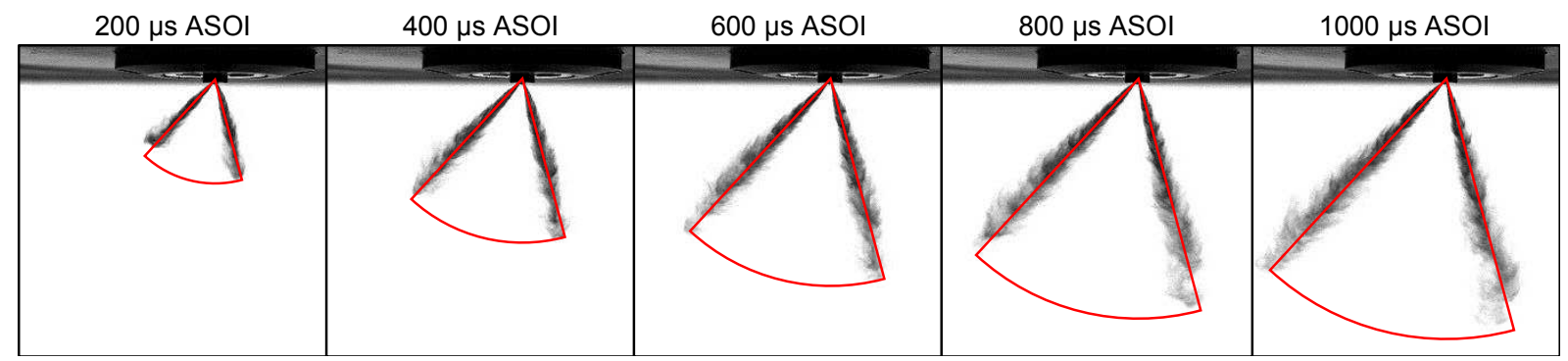

Figure 3. Shadowgraphy at five timesteps

Then, the simulation results of the segment model are compared with LDM measurements of the two single plumes. Figure 4 shows the spray in the injector nearfield after an injection duration of approximately $1 \mathrm{~ms}$. The fuel is represented by isosurfaces with a constant volume fraction of 0.1 colored with the corresponding velocity. The red lines represents the plume opening angle $\beta$ closest to the counterbore exit plane. In Table 2 the penetration after $600 \mu \mathrm{s}$ is linked to the spray opening angle, confirming the expected relationship of a declining penetration with increasing opening angle. Furthermore, the direction of the simulated spray plumes match the measured ones, see Figure 4. It is worth noting that the RANS simulation shows a starting breakup, so that also the onset the atomization process is approximately covered. Anyhow, the focus of this paper will be limited to the spray opening angle $\beta$.

Figure 5 shows contours of fuel volume fraction as well as velocity vectors to compare the spray direction and spray opening angle $\beta$. With help of the nozzle flow data the measured and simulated spray opening angles $\beta$ can be analyzed, see Table 2. The simulation is able to predict the opening angle for both holes at the counterbore exit planes in close agreement with the measurement. The simulated $\beta$-angles are for both holes approximately two degree too low, which can also be slightly seen in Figure 4. However, it is crucial to note that SH2 has, compared to $\mathrm{SH} 1$, an almost two degree higher opening angle in the simulation as well as in the measurements. Hence, the 


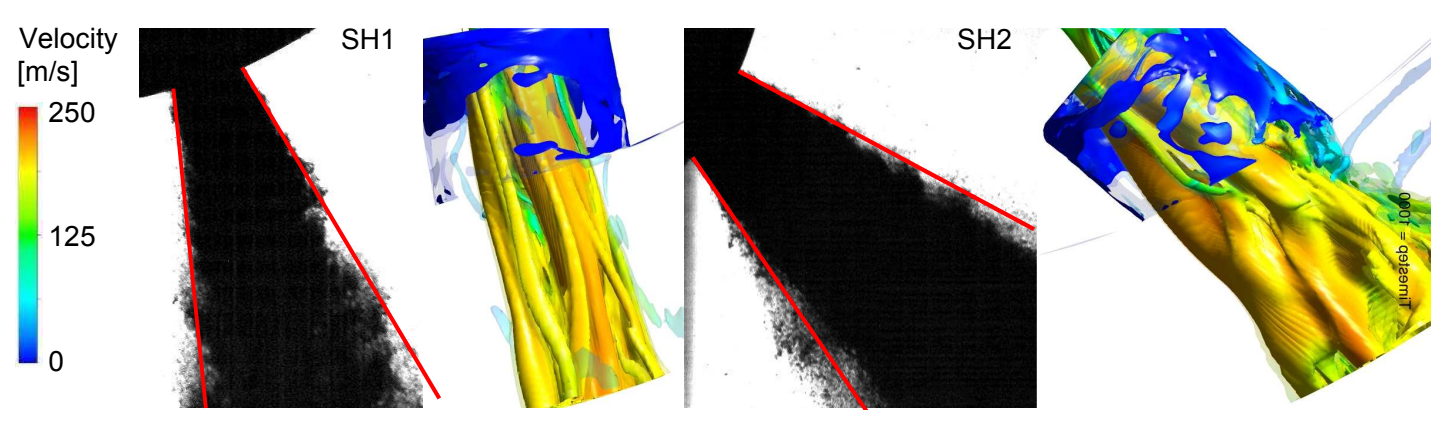

Figure 4. LDM vs. simulated isosurfaces

correlation between spray opening angle and penetration length is found identically in simulation and experiment. To limit computational effort, the spray opening angle at the spray hole outlet will be used as an objective for the optimization tasks. At this plane the simulated values are higher, as the spray guidance of the counterbore walls is missing, see Table 2. Hence, the described tendency is even more intense at this plane, leading to a high $\beta$-angle of $42.94^{\circ}$ for $\mathrm{SH} 2$ compared to $32.10^{\circ}$ for $\mathrm{SH} 1$.

Table 2. Correlations between measurements, objective $\beta$ and inner nozzle flow data

\begin{tabular}{lc|cc|cc}
\hline \multicolumn{2}{c|}{ Geometry } & \multicolumn{2}{c|}{ Measured } & \multicolumn{2}{c}{ Simulation at Outlet of } \\
referred as & $\alpha$ & Penetration $(600 \mu s)$ & $\beta$ & $\beta$ CB & $\beta$ SH \\
\hline SH1 & $10^{\circ}$ & $58.13 \mathrm{~mm}$ & $24.55^{\circ}$ & $22.82^{\circ}$ & $32.10^{\circ}$ \\
SH2 & $50^{\circ}$ & $56.40 \mathrm{~mm}$ & $26.68^{\circ}$ & $24.96^{\circ}$ & $42.94^{\circ}$
\end{tabular}

\section{Contour: \\ 1.0 \\ 0.5 \\ 0.0}

Fuel Volume

Fraction [-]
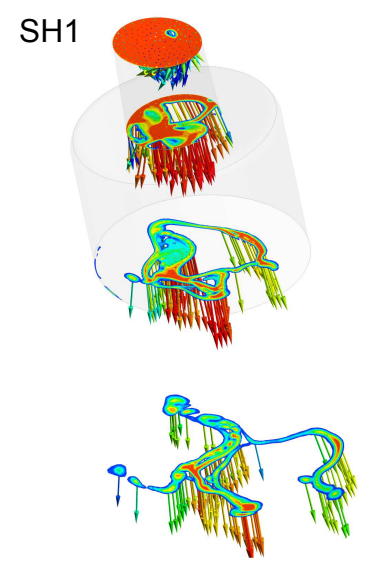

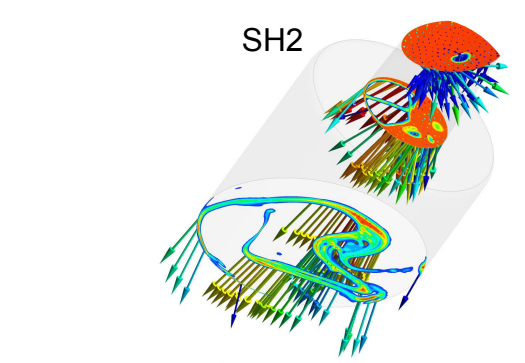

Vector: Velocity $[\mathrm{m} / \mathrm{s}]$

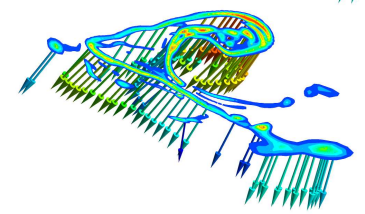

Figure 5. Distribution of fuel volume fraction and velocity vectors

Finally the validation shows that the simulation setup is able to capture the nozzle flow characteristics. A correlation between the objective (low spray penetration) and the spray opening angle $\beta$ can be clearly seen. The values of the predicted spray opening angle $\beta$ at counterbore outlet are in close agreement to the measured angles. At spray hole exit, much higher values are found, but the proper tendency can be clearly identified. Thus, the $\beta$ angle at spray hole exit can be used for optimization.

\section{Metamodeling and Optimization}

The aim of a multi-objective problem is to improve simultaneously the values of all, at least two, objectives, without violating any predefined constraint. A minimization problem can, without any loss of generality, be defined as in equation 1 ,

$$
\begin{aligned}
& \min _{\mathbf{x} \in \mathbb{R}^{n}} y(\mathbf{x}), \text { with } y(\mathbf{x})=\left(f_{1}(\mathbf{x}), f_{2}(\mathbf{x}), \ldots, f_{m}(\mathbf{x})\right), m=1,2, \ldots, M \\
& \text { subject to } g_{k}(\mathbf{x}) \leq 0, k=1,2, \ldots, K \\
& h_{l}(\mathbf{x})=0, l=1,2, \ldots, L \\
& x_{i}^{l} \leq x_{i} \leq x_{i}^{u}, i=1,2, \ldots, N
\end{aligned}
$$

where $\mathbf{x}$ is the $N$-dimensional vector of design variables, $f_{m}(\mathbf{x})$ are the $M$ objective functions, $g_{k}(\mathbf{x})$ are the $K$ inequality constraints and $h_{l}(\mathbf{x})$ are the $L$ equality constraints. In the present study only inequality constraints are 
used $(L=0)$ so that the feasible design space $\mathbb{X}$ must satisfy the $K$ inequality constraints, lay in between the lower $x_{i}^{l}$ and upper $x_{i}^{u}$ parameter boundaries and be part of $\mathbb{R}^{n}\left(\mathbf{x}^{n} \in \mathbb{X}^{n} \in \mathbb{R}^{n}\right)$. The definition of the multi-objective minimization problem is given in equation 2 .

$$
\text { (I) }: y(\mathbf{x}) \stackrel{\mathbf{x}}{\rightarrow} \min , \text { with } \mathbf{x}^{n} \in \mathbb{X}^{n}
$$

As the selected objectives might be incompatible with each other, a global optimum often does not exist for a multi-objective problem. Hence, comparing two designs and deciding which one is better can become difficult or even impossible. Pareto-dominance is a sorting method that allows identification of the best design from a set of solutions. This method delivers an a posteriori analysis and is defined as follows:

$$
\hat{\mathbf{x}} \prec \mathbf{x} \Leftrightarrow\left\{\begin{array}{l}
\forall i \in[1 \ldots M]: f_{i}(\hat{\mathbf{x}}) \leq f_{i}(\mathbf{x}) \\
\exists j \in[1 \ldots M]: f_{j}(\hat{\mathbf{x}})<f_{j}(\mathbf{x})
\end{array}\right.
$$

Pareto-dominance in equation 3 states that design variable $\hat{\mathbf{x}}$ dominates design variable $\mathbf{x}$ if $f(\hat{\mathbf{x}})$ is not worse for each objective $i$ of $f(\mathbf{x})$ and is at least better in one objective $f_{j}(\mathbf{x})$. A dominant design $\hat{\mathbf{x}}$ is always the preferred solution. With this analysis, the optimization process delivers the Pareto front, i.e. a set of non-dominated solutions, defined as:

$$
P(\mathbb{X}):=\{\mathbf{x} \in \mathbb{X} \mid \nexists \hat{\mathbf{x}} \in \mathbb{X}: \hat{\mathbf{x}} \prec \mathbf{x}\}
$$

Equation 4 states that all Pareto-optimal solutions are of the same quality. The final goal is to find as many as possible Pareto-optimal solutions in one single run [5]. Except for some simple analytical cases the exact Pareto front cannot be reached, so that iterative methods are required to approximate the front $[3,13]$. Genetic Algorithms are easy to use, widely applicable, and deliver a global optimum. They are therefore broadly used as search and optimization tools [4]. GA use the principle of natural selection including semi-stochastic processes. There are many common GA available [9]. NSGA-II is one of the most well-known approach for multi objective optimization problems [5]. The detailed implementation of the NSGA-II algorithm in OPAL++, e.g. dominance, constraint handling and validity, can be found in [2].

To initialize the population various DoE approaches exist $[1,12]$. Based on the first generation a new set of individuals, the offspring, are generated using the genetic operators selection, crossover and mutation. During the iterative optimization process the GA prefer the individuals with high performance for further genetic operations. Therefore, the new offspring will drift iteratively towards the Pareto front.

Table 3. Optimization settings

\begin{tabular}{c|cccc|cc}
\hline & \multicolumn{4}{|c|}{ Design variable } & \multicolumn{2}{c}{ Objectives at SH Outlet } \\
& $c b_{d}$ & $s h_{\text {con }}$ & $s h_{l}$ & $w t$ & $\beta$ & TKE \\
\hline Lower bound & $300 \mu \mathrm{m}$ & $0^{\circ}$ & $125 \mu \mathrm{m}$ & $300 \mu \mathrm{m}$ & - & - \\
Upper bound & $550 \mu \mathrm{m}$ & $25^{\circ}$ & $250 \mu \mathrm{m}$ & $600 \mu \mathrm{m}$ & $\max$ & $\max$
\end{tabular}

Table 3 represents the four input parameters with their ranges and the two objectives: maximize TKE and $\beta$ at the spray hole outlet. Furthermore, a minimum counterbore length is set with an inequality constraint $g_{1}(\mathbf{x})$. To construct a polynomial approximation for the described problem a DoE of size 142 is first generated using Sobol sampling, a quasi-random sequence delivering a uniform distribution. This method is recommended for limited sample sizes, as here [1]. Based on the DoE an Iterative Reweighted Least Square (IR_LSQ), a global polynomial regression method of total order three is applied as a Response Surface having 35 coefficients $c_{m}$. The estimated value $s$ of the IR_LSQ is given by:

$$
\begin{array}{r}
s(\mathbf{x})=p^{T}(\mathbf{x}) c_{m} \\
\text { with } p^{T}(\mathbf{x})=\left[p_{1}(\mathbf{x}), p_{2}(\mathbf{x}), \ldots p_{m}(\mathbf{x})\right]
\end{array}
$$

The unknown coefficients $c_{m}$ are iteratively determined by minimizing the weighted least square errors between predicted values $s$ and the simulated values $Y$ at the $N$ sample points:

$$
\sum_{i=1}^{N}\left[p^{T}\left(\mathbf{x}_{i}\right) c_{m}-Y\right]^{2} \stackrel{c}{\rightarrow} \min
$$

Due to software failures, poor mesh quality or constraint violations 37 design have been found to be invalid, so that in total 105 designs have been evaluated on a High Performance Computing (HPC) cluster. In Figure 6 a poor fit for the objective TKE is seen with a R-square value of only 0.34 . This result could probably be improved by adding more coefficients to the IR_LSQ model [6], which is here impossible due to the high computational effort of each single evaluation. Especially the prediction of the individual with a simulated TKE of approx. $300 \mathrm{~m}^{2} / \mathrm{s}^{2}$ is poor, showing that the metamodel does not properly predict the region of interest (high TKE values). Fortunately, the predicted values for the spray opening angle $\beta$ show over the whole range an excellent agreement with the simulated ones, see Figure 7. 
In Figure 8 the 3D-response for TKE and in Figure 9 the response for $\beta$ over the spray hole conicity $s h_{\text {con }}$ and wall thickness $w t$ of the nozzle seat are shown. The two normalized 3D-responses are only valid for selected values of counterbore diameter and spray hole length $\left(c b_{d}=400 \mu \mathrm{m}, s h_{l}=200 \mu \mathrm{m}\right)$. For this configuration the spray opening angle $\beta$ is dominated by the spray hole conicity $s h_{c o n}$, leading to a higher spray opening angle $\beta$ when decreasing spray hole conicity $s h_{c o n}$.
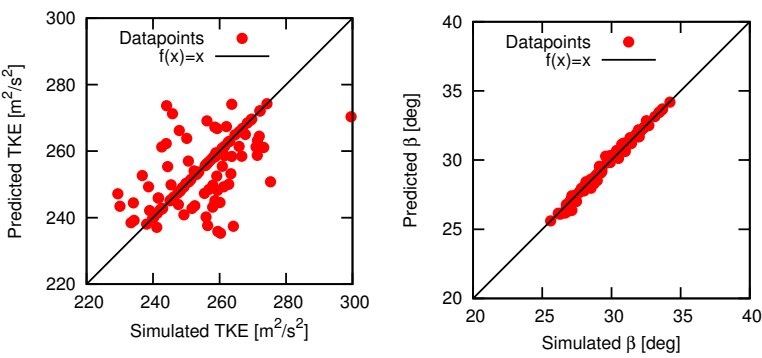

Figure 6. Metamodeling TKE
Figure 7. Metamodeling for $\beta$

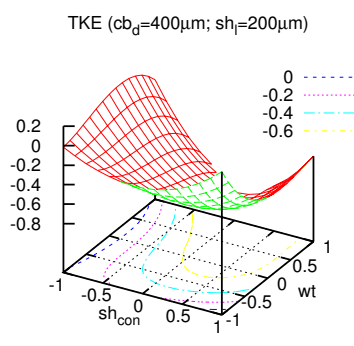

Figure 8. Reponse for TKE

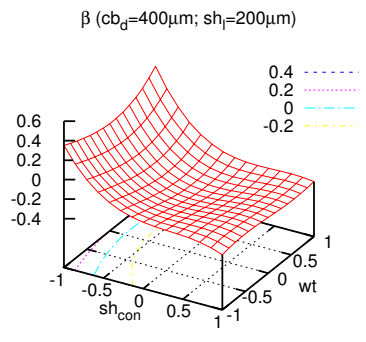

Figure 9. Response for $\beta$

The two IR_LSQ models are then used in a metamodel-driven optimization, which evaluates a parameter set by using two algebraic equations. This fast evaluation allows a NSGA-II multi-objective optimization with a large population size of 200 for 50 generations. Figure 10 shows the distribution of the DoE, the Pareto front of the first and of the last $\left(50^{\text {th }}\right)$, generation. Additionally, three design points (ID1 - ID3), at the most meaningful positions of the Pareto front, are selected for validation; the corresponding CFD results are shown with colored triangles. In agreement with Figure 7, the prediction of $\beta$ obtained by CFD agrees with the metamodel; the triangles stay roughly at the same height in the diagram; however, CFD leads to a much different value of TKE, so that the triangles are widely shifted to the left side.

Figure 11 links the objectives of the three selected Pareto-optimal solutions with the design variables. The TKE at spray hole outlet plane as well as velocity vectors are also shown. All configurations correspond to the lowest possible counterbore diameter and spray hole length. The high difference in spray opening angle $\beta$ between ID1 and the rest of the Pareto-optimal solutions is due to the small spray hole conicity and the highest possible wall thickness. ID2 and ID3 correspond to small geometry changes in wall thickness and spray hole conicity. Therefore, the simulated results from ID2 and ID3 only differ slightly from each other. It appears that TKE metamodel shows excessive sensitivity, leading to poor prediction accuracy, especially in the region of high TKE values.

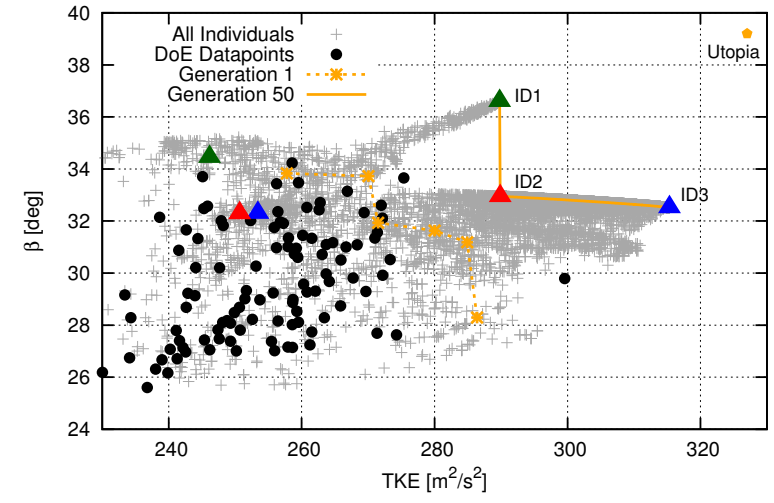

Figure 10. Pareto front for the metamodel-based NSGA II
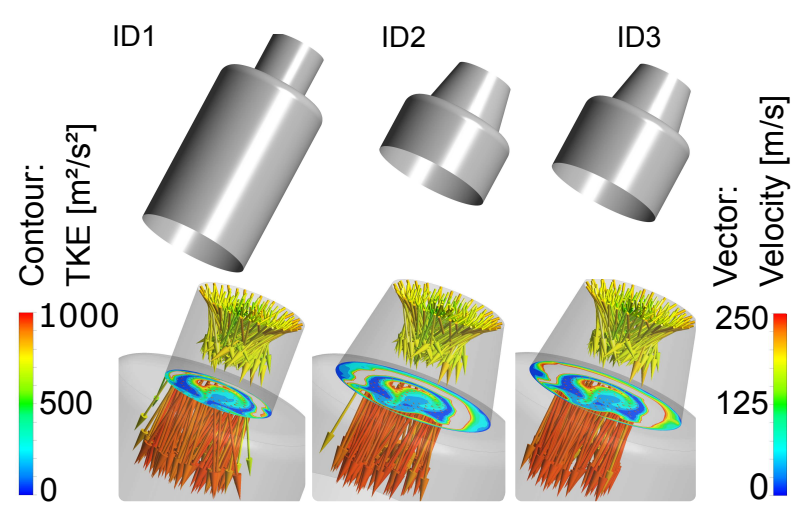

Figure 11. Geometry and flow of Pareto-optimal designs

In order to check how the true Pareto front looks like, a direct multi objective optimization with the NSGA-II algorithm has been carried out. Unfortunately, due to computational cost, it involves only a population size of 48 . Figure 12 depicts the DoE and the Pareto front of the first and last $\left(10^{\text {th }}\right)$ generation. A design improvement can clearly be seen as the whole Pareto front moves towards the top-right corner. Again, three meaningful optimal individuals have been selected for a detailed analysis, illustrated in Figure 13.

A low spray hole conicity shows a positive effect on TKE and on $\beta$, so that all three designs correspond to minimal conicity. A broader range for this parameter could deliver even better designs. The remaining three design variables show large differences within the Pareto-optimal solutions. For the spray hole length two contradictory trends can be observed for the objectives: The higher the spray hole length, the higher the TKE; the lower the spray hole length, the higher the spray opening angle $\beta$. ID1 has the maximum TKE with an intermediate counterbore diameter, a maximum spray hole length and a low wall thickness resulting in a very short counterbore length. On the opposite, ID5 shows the highest simulated value in $\beta$, but a low TKE value.

Like in the validation section, the flow is investigated with the help of contours representing the fuel volume fraction 


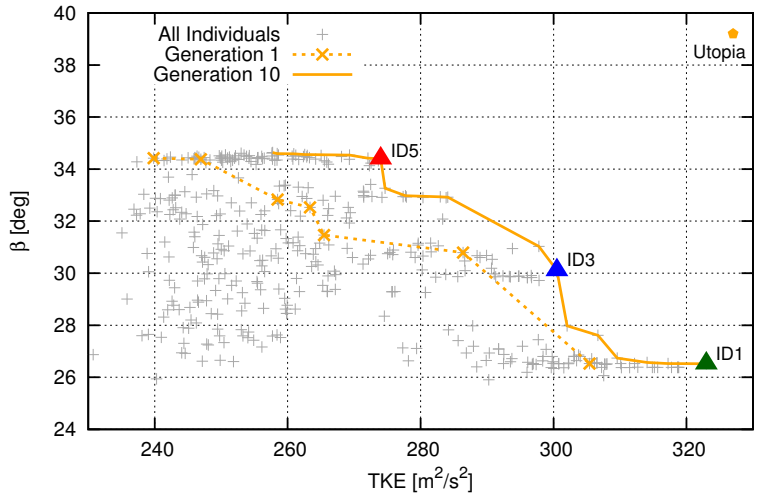

Figure 12. Pareto front for the direct NSGA II

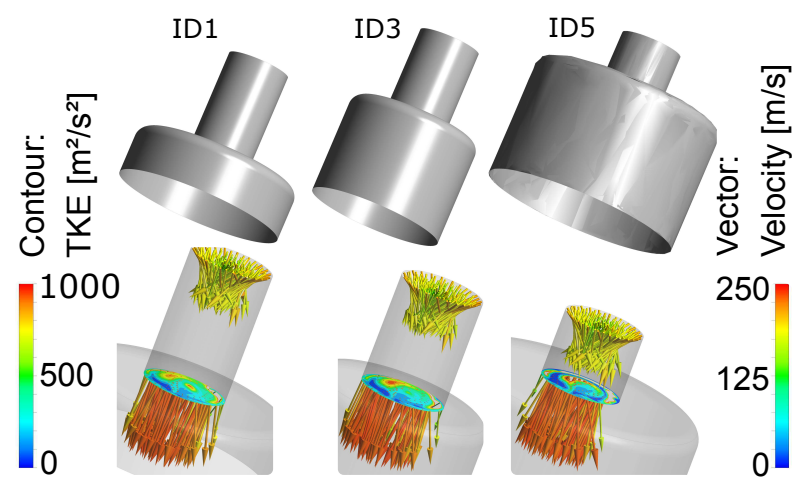

Figure 13. Geometry and flow of Pareto-optimal designs

and velocity vectors (Figure 14). The volume fractions at the bottom show the distributions at the opening boundary of the segment model. Looking at the velocity vectors it is confirmed that a longer spray hole leads to a lower $\beta$ angle. Therefore, ID5 shows the widest spread of the spray plume. The trends detected by the direct optimization can also be seen further downstream, e.g. at the counterbore outlet and at the boundary condition of the simulation domain.

\section{Contour: \\ Fuel Volume \\ Fraction [-]}

1.0
0.5
0.0

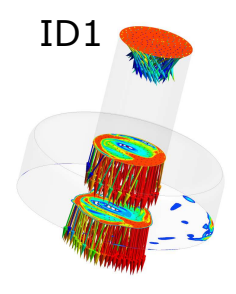

ID1

(n)

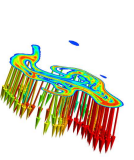

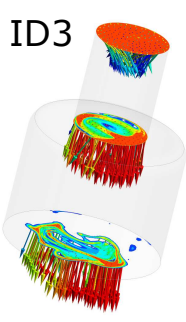

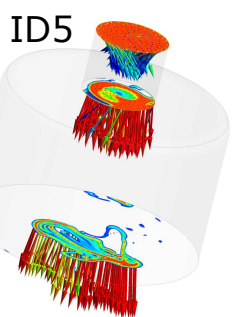

Vector:

Velocity $[\mathrm{m} / \mathrm{s}]$

Figure 14. Distribution of Fuel Volume Fraction and velocity vectors

\section{Conclusions}

The RANS simulation setup of a segment model has the capability to reveal the main flow characteristics for a GDI inner nozzle flow. A comparison with LDM and shadowgraphy measurements show correct trends concerning spray direction, spray opening angle and distribution of the plume at counterbore outlet. First correlations between inner nozzle flow and penetration length have been derived and used as objective functions for a multi-objective optimization. Reaching a low penetration while conserving a high turbulent breakup should be obtained thanks to a high TKE and a high spray opening angle at the spray hole outlet.

An optimization setup with four design variables has been presented. Response surfaces (IR_LSQ of order three) for a Sobol-sampled DoE containing 105 valid design points have been analyzed leading to following conclusions:

- The metamodel for the spray opening angle $\beta$ shows promising results and is suitable for optimization.

- The TKE-metamodel is not accurate enough to capture the influence of geometry changes.

Hence, selected Pareto-optimal solutions of the metamodel-based optimization show high deviations in TKE when comparing with CFD. For comparison with this fast metamodel optimization, a computationally challenging direct optimization was carried out using NSGA2 within approximately 250,000 CPU hours. After 10 generations with a population size of 48 , considerably improved designs can be obtained. The two most noticeable results affecting nozzle design are:

1. A low spray hole conicity has a positive effect for both objectives. Therefore, all the optimal solutions nearly share the lowest possible spray hole conicity.

2. The longer the spray hole length, the lower the spray opening angle, but the higher the TKE. 
The use of GA for nozzle seat optimization will be pursued. The parameter range for the investigated variables will be increased towards manufacturing constraints. The influence of turbulence modelling on complex nozzle flows will be further investigated by comparing the results of RANS with LES (Large-Eddy-Simulations). Using a second validation case, the prediction reliability of the simulation model regarding spray plume penetration will be verified, increasing confidence in the presented approach.

\section{Acknowledgements}

The authors would like to thank Dipl.-Ing. M. Lausch for his support regarding the simulation domain and setup. Furthermore we would like to thank Dr. P. Leick for providing the shadowgraphy and LDM measurements.

\section{Nomenclature}

$\begin{array}{ll}\text { DoE } & \text { Design-of-Experience } \\ \text { LDM } & \text { Long-Distance-Microscope } \\ \text { GDI } & \text { Gasoline-Direct-Injection } \\ \text { CFD } & \text { Computational Fluid Dynamics } \\ \text { CFD-O } & \text { CFD-Optimization } \\ \text { GA } & \text { Genetic Algorithm } \\ \text { RSM } & \text { Response Surface Method } \\ \text { RANS } & \text { Reynolds Averaged Navier Stokes } \\ \text { LES } & \text { Large-Eddy-Simulation } \\ \text { IR_LSQ } & \text { Iterative Reweighted Least Square }\end{array}$

$\begin{array}{ll}\text { HPC } & \text { High Performance Computing } \\ \text { NSGA } & \text { Non-Sorting Genetic Algorithm } \\ \text { TKE } & \text { Turbulent Kinetic Energy }\left[\mathrm{m}^{2} \mathrm{~s}^{-2}\right] \\ c b_{d} & \text { counterbore diameter }[\mu \mathrm{m}] \\ s h_{d} & \text { spray hole diameter }[\mu \mathrm{m}] \\ s h_{\text {con }} & \text { spray hole conicity [deg] } \\ s h_{l} & \text { spray hole length }[\mu \mathrm{m}] \\ w t & \text { wall thickness }[\mu \mathrm{m}] \\ \alpha & \text { inclination angle of spray hole }[\mathrm{deg}] \\ \beta & \text { spray plume opening angle [deg] }\end{array}$

\section{References}

[1] Burhenne, S., Jacob, D., Henze, G., Nov. 14.-16. 2011, 12th Conference of International Building Performance Simulation Association.

[2] Daróczy, L., 2016, "Practical issues in the optimization of CFD based engineering problems". PhD thesis, University Magdeburg.

[3] Daróczy, L., Janiga, G., Thévenin, D., 2014, Energy, 65, pp. 364-373.

[4] Deb, K., 1999, Sadhana, 24 (4-5), pp. 293-315.

[5] Deb, K., Pratap, A., Agarwal, S., Meyarivan, T., 2002, IEEE Transactions on Evolutionary Computation, 6 (2), pp. 182-197.

[6] Ender, T., Balestrini-Robinson, S., 2015, "Modeling and Simulation in the Systems Engineering Life Cycle". Springer

[7] Gen, M., Cheng, R., 2000, "Genetic Algorithms and Engineering Optimization". John Wiley and Sons.

[8] Janiga, G., Thévenin, D., 2008, "Optimization and Computational Fluid Dynamics". Springer.

[9] Konak, A., Coit, D., Smith, A. E., 2006, Reliability Engineering \& System Safety, 91 (9), pp. 992-1007.

[10] Köpple, F., Jochmann, P., Kufferath, A., Bargende, M., 2013, SAE International Journal of Engines, 6, pp. 911-925.

[11] Kufferath, A., Wiese, W., Samenfink, W., Dageförde, H., Knorsch, T., Jochmann, P., Mar. 10.-11. 2015, Fuel Systems for IC Engines.

[12] Macdonald, I. A., July 27.-30. 2009, Eleventh International IBPSA Conference.

[13] Marler, T. R., Arora, J. S., 2004, Structural and Multidisciplinary Optimization, 26 (6), pp. 369-395.

[14] Matsumoto, A., Xie, X., Zheng, Yi., Lai, M., Moore, W., May 16.-19. 2010, 22nd Annual Conference on Liquid Atomization and Spray Systems.

[15] Mitroglou, N., Nouri, J. M., Gavaises, M., Arcoumanis, C., 2006, International Journal of Engine Research, 7 (3), pp. 255-270.

[16] Myer, R. H., Montgomery, D. C., 2002, "Response Surface Methodology: Process and Product Optimization Using Designed Experiments". John Wiley and Sons.

[17] Payri, R., Viera, J., Gopalakrishnan, V., Szymkowicz, P. G., 2016, Fuel, 183, pp. 20-33.

[18] Sacks, J., Welch, W. J., Mitchell, T. J., Wynn, H. P., 1989, Statistical science, 4 (4), pp. 409-423.

[19] Simpson, T., Mistree, F., Korte, J., Mauery, T., 1998, 7th AIAA/USAF/NASA/ISSMO Symposium on Multidisciplinary Analysis and Optimization.

[20] Tu, P. W., Xu, H., Srivastava, D. K., Dean, K., Jing, D., Cao, L., Weall, A., Venus, J. K., Sep. 1. 2015, JSAE/SAE 2015 International Powertrains, Fuels \& Lubricants Meeting.

[21] Wiese, W., Kufferath, A., Storch, A., Rogler, P., Feb. 24.-25. 2015, 2. Internationaler Motorenkongress.

[22] Zhao, F.-Q., Yoo, J.-H, Liu, Y., Lai, M.-C., Oct. 1. 1996, 1996 SAE International Fall Fuels and Lubricants Meeting and Exhibition.

[23] Robert Bosch GmbH, http://www.bosch-mediaspace.de/ ([cit. 2017-02-22]).

[24] ANSYS Inc., CFX Solver Theory Guide, http://www . ansys . com/ ([cit. 2017-02-28]). 